\title{
Mismatch repair-deficient hormone receptor-positive breast cancers: Biology and pathological characterization
}

\author{
Elham Sajjadi ${ }^{1,2+}$, Konstantinos Venetis ${ }^{1,2 \dagger}$, Roberto Piciotti ${ }^{1,2}$, Marco Invernizzi ${ }^{3}$, Elena Guerini-Rocco ${ }^{1,2}$, \\ Svasti Haricharan ${ }^{4}$ and Nicola Fusco $1,2^{*}$ (i)
}

\begin{abstract}
The clinical outcome of patients with a diagnosis of hormone receptor (HR) + breast cancer has improved remarkably since the arrival of endocrine therapy. Yet, resistance to standard treatments is a major clinical challenge for breast cancer specialists and a life-threatening condition for the patients. In breast cancer, mismatch repair (MMR) status assessment has been demonstrated to be clinically relevant not only in terms of screening for inherited conditions such as Lynch syndrome, but also for prognostication, selection for immunotherapy, and early identification of therapy resistance. Peculiar traits characterize the MMR biology in HR+breast cancers compared to other cancer types. In these tumors, MMR genetic alterations are relatively rare, occurring in $\sim 3 \%$ of cases. On the other hand, modifications at the protein level can be observed also in the absence of gene alterations and vice versa. In HR+ breast cancers, the prognostic role of MMR deficiency has been confirmed by several studies, but its predictive value remains a matter of controversy. The characterization of MMR status in these patients is troubled by the lack of tumor-specific guidelines and/or companion diagnostic tests. For this reason, precise identification of MMR-deficient breast cancers can be problematic. A deeper understanding of the MMR biology and clinical actionability in HR+ breast cancer may light the path to effective tumor-specific diagnostic tools. For a precise MMR status profiling, the specific strengths and limitations of the available technologies should be taken into consideration. This article aims at providing a comprehensive overview of the current state of knowledge of MMR alterations in HR+ breast cancer. The available armamentarium for MMR testing in these tumors is also examined along with possible strategies for a tailored pathological characterization.
\end{abstract}

Keywords: Breast cancer, Mismatch repair, DNA repair, Immune checkpoint, Immunotherapy

\section{Background}

Every nine seconds, worldwide, a woman is diagnosed with breast cancer, which is by far the most prevalent female tumor and a leading cause of cancer-related death [1]. Approximately two-thirds of these patients have a hormone receptor $(\mathrm{HR})+$ disease, meaning that the

\footnotetext{
${ }^{*}$ Correspondence: nicola.fusco@unimi.it

${ }^{\dagger}$ Elham Sajjadi and Konstantinos Venetis contributed equally to this work

1 Division of Pathology, IEO, European Institute of Oncology IRCCS, University of Milan, Via Giuseppe Ripamonti 435, 20141 Milan, Italy

Full list of author information is available at the end of the article
}

tumor expresses estrogen and/or progesterone receptors [2]. Endocrine therapy (ET), alone or in combination with chemotherapy, and/or targeted therapies, represents the medical treatment backbone in this setting [3]. A significant percentage of patients with $\mathrm{HR}+$ breast cancer, however, eventually develop therapy resistance due to several mechanisms, including tumor immune microenvironment modulation, and mismatch repair (MMR) downregulation [4-7].

The MMR system is an innate form of defense against DNA base mispairing that is essential to human

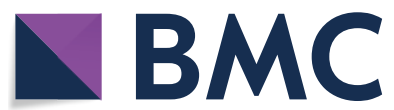

(c) The Author(s) 2021. This article is licensed under a Creative Commons Attribution 4.0 International License, which permits use, sharing, adaptation, distribution and reproduction in any medium or format, as long as you give appropriate credit to the original author(s) and the source, provide a link to the Creative Commons licence, and indicate if changes were made. The images or other third party material in this article are included in the article's Creative Commons licence, unless indicated otherwise in a credit line to the material. If material is not included in the article's Creative Commons licence and your intended use is not permitted by statutory regulation or exceeds the permitted use, you will need to obtain permission directly from the copyright holder. To view a copy of this licence, visit http://creativeco mmons.org/licenses/by/4.0/. The Creative Commons Public Domain Dedication waiver (http://creativecommons.org/publicdomain/ zero/1.0/) applies to the data made available in this article, unless otherwise stated in a credit line to the data. 
physiology [8]. This highly sophisticated cellular mechanism is modulated by both environmental stimuli and internal processes, resulting in the preservation of the DNA status quo [9]. Detrimental modifications in the MMR complex cause genome instability, which is a precondition for cancer to arise [8,9]. During the past few years, the clinical actionability of MMR alterations has become increasingly important in breast cancer, not only in terms of screening for inherited conditions, but also for patients' prognostication, prediction of immune checkpoint blockers (ICB) efficacy, and early identification of resistance to therapies $[10,11]$.

Intrinsic differences seem to characterize the frequency, types, and patterns of MMR alterations in $\mathrm{HR}+$ breast cancers compared to HR- breast cancers and other cancer types [12]. Regrettably, our understanding of the biology that governs the MMR machinery and its clinical actionability in $\mathrm{HR}+$ breast cancer remains incomplete. The substantial lack of tumor-specific guidelines and/or companion diagnostic tests (CDx), further troubles the pathological identification of MMR-deficient (dMMR) cases. In this review, we provide an overview of the specific role of MMR in HR+breast cancer and discuss the currently available testing strategies for the precise identification of these patients.

\section{The mismatch repair system machinery}

Genetic mutations are natural events during DNA replication [13]. Despite not all DNA base mismatches are detrimental, their thorough correction prevents pathogenic mutations from being passed through the cell line [9]. In this respect, the MMR system plays a key role in maintaining genomic integrity and cell homeostasis [14]. The foremost MMR components are mutL homolog 1 (MLH1), mutS homolog 2 (MSH2), mutS homolog 6
(MSH6), mutS homolog 3 (MSH3), post-meiotic segregation increased 2 (PMS2), proliferating cellular nuclear antigen (PCNA) and EXO1 $3^{\prime} \rightarrow 5^{\prime}$ exonucleases [8, 9, 15]. In particular, $\mathrm{MSH} 2$ together with $\mathrm{MSH} 6$ or $\mathrm{MSH} 3$ compose the MutS $\alpha$ and MutS $\beta$ heterodimers, respectively, while MLH1 heterodimerizes together with PMS2 to form MutL $\alpha$ [16]. These complexes interact with each other to regulate the recognition and cleavage of incorrect base insertions (Fig. 1) [17]. Malfunction of the MMR system may be responsible for DNA instability, thus promoting tumorigenesis, tumor progression, and resistance to therapies $[18,19]$. A collateral phenomenon of MMR-induced genomic instability is represented by microsatellite instability (MSI) [20, 21]. Microsatellites are short tandem repeating sequences of 1-6 nucleotides widely distributed over the DNA, mainly located near the coding region and the ends of chromosomes [22, 23]. Pioneer studies provided evidence suggesting that MSI could be considered, for clinical purposes, as a proxy of the overall genome instability generated by MMR deficiency [24]. Given the elevated frequency of MSI-high (MSI-H) status in dMMR tumors, MMR protein expression and MSI have been historically considered reliable, cost-effective, and (to some extent) interchangeable biomarkers in oncology [25].

\section{Mismatch repair alterations in $\mathrm{HR}+$ breast cancer Frequency and specific pathways involved}

Breast cancers may harbor a wide spectrum of scars in the MMR system, including gene mutations, promoter hypermethylation, and downregulation of RNA levels, as well as alterations to cellular localization of the protein complexes [21, 26-30]. Gene signatures of MMR perturbation have been described in approximately $3 \%$ of these patients, while impaired expression of the MMR proteins

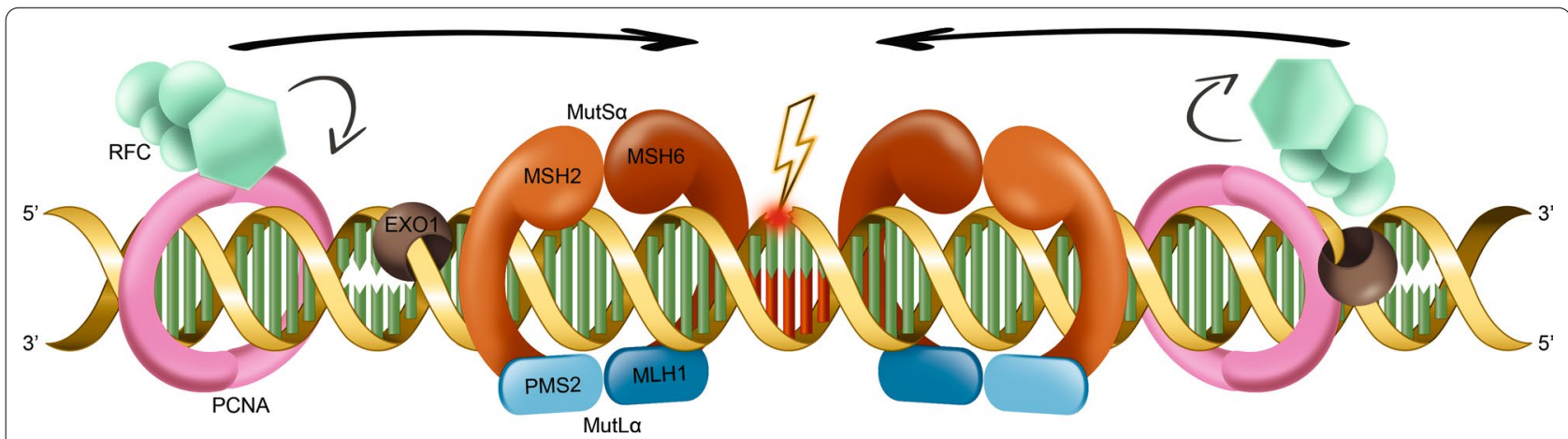

Fig. 1 Schematic representation of the MMR system maincomponents. MutSa complex (heterodimer MSH2-MSH6) initiates repairsignaling by recognizing the mismatch (thunder). Then, MutLa (heterodimerMLH1-PMS2) is recruited, generating a ternary complex that mediates thedownstream processes. Proliferating cell nuclear antigen (PCNA) and replicationfactor C (RFC) are subsequently activated by MutS. In particular, RFC loadsPCNA which is directly implicated in the excision repair and DNA synthesisprocess. The assembly will initiate endonuclease activity of PMS2 which createssingle-strand breaks close to the mismatch and allows for the removal of thewrong-inserted base by exonuclease 1 (EXO1) 
seems to be more frequent [31]. Several studies confirmed that MMR deficiency is significantly associated with an increased risk of death in breast cancers, particularly in the HR+ group [21, 29, 32]. Despite this recognized prognostic role, the predictive value of MMR alterations is still controversial in these tumors [33]. Hence, only a few breast cancers were included in the basket trials that led to the histology-agnostic approval of ICB in the presence of MMR deficiency [34]. Of note, none of these patients had a diagnosis of HR+breast cancer (Fig. 2). Another debated aspect of $\mathrm{dMMR}$ HR+ breast cancers is linked to the assumption that the sensitivity to ICB is mainly related to the adaptative immune response against neoantigens generated by super-mutator cancers [35]. Albeit fitting to some tumor types (e.g. endometrial and colorectal cancers), this model shows certain limitations in $\mathrm{HR}+$ breast neoplasms [36]. Indeed, the tumor mutation burden (TMB) is typically lower in $\mathrm{dMMR} \mathrm{HR}+$ breast cancers compared to HR-/HER2- and HER2+tumors $[37,38]$.

In HR+breast cancer, there is a gap of frequency between MMR gene and protein deficiency, where the latter phenomenon is more common than the former

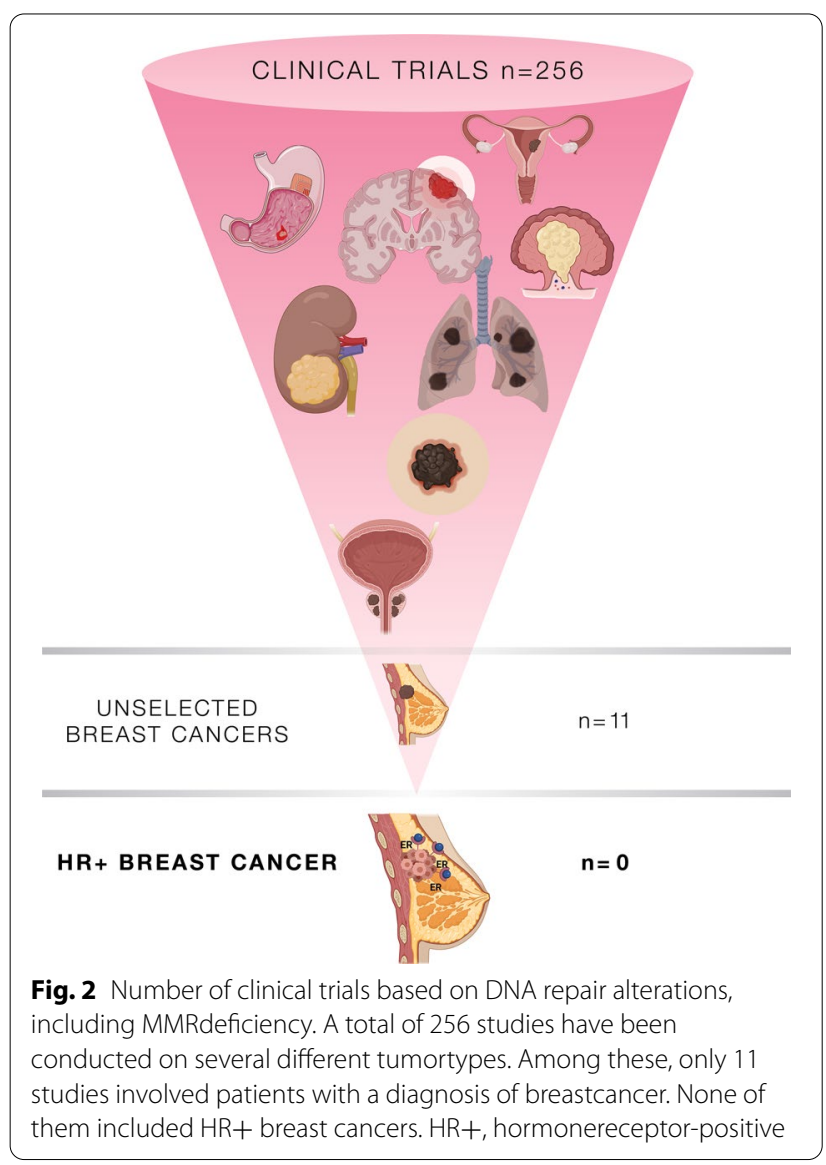

[12]. Finding a sound explanation for this remains a subject of controversy, with some authors pointing out suboptimal standard operating procedures in MMR testing and others positing intrinsic biological traits of dMMR $\mathrm{HR}+$ breast cancers [39]. In support of this hypothesis, there is the observation of distinct dMMR phenotypes in HR+breast cancers. From the proteomic dimension, these tumors are more likely to show a single-marker impairment, preferentially in the MutL $\alpha$ component, unlike in other tumor types (e.g. endometrial and colorectal cancer) that usually show proteins-pair loss $[7,12$, 40]. Taken together, the loss of expression at a singleprotein level is more commonly observed in $\mathrm{HR}+$ breast cancer compared to HR- (Table 1). More in detail, loss of MLH1 and PMS2 is reported in 7 and $3 \%$ of cases, respectively [30]. The analysis of publicly available nextgeneration sequencing (NGS) data confirms this propensity, albeit with the aforementioned lower frequency of events, as shown in Fig. 3. Another peculiar trait of breast cancer is represented by the heterogeneous distribution of the MMR+ neoplastic cells. Hence, in more than $13 \%$ HR + breast cancers (both HER2+ and HER2-), the MMR proteins are heterogeneously expressed inside the tumor without a preferential distribution pattern [21, 41]. The therapeutic implications of this intra-tumor heterogeneity might be critical in regulating intrinsic ET resistance. While the sensitivity of MutL-deficient HR+ breast cancers to ET is decreased, MutS-deficient cases show the contrary [7]. This sort of gene selectivity in dMMR breast cancers might be controlled by cellular signaling pathways that have not been clarified yet. However, the association of MLH1 with ATM activation and the formation of MSH2 complex with ATR/Chk1 activation are events that potentially play a role in these variable ET sensitivities [7, 42]. Of note, a significant portion of $\mathrm{HR}+$ breast cancers shows dysregulation of the phosphoinositide 3 kinase (PI3K)/AKT/mammalian target of rapamycin (mTOR) signaling, which is also the main contributor to ET resistance [43, 44]. Furthermore, actionable co-alterations in PIK3CA and DNA repair genes might lead to the activation of the human leukocyte antigen (HLA) molecules due to increased TMB and subsequent neoantigens production, particularly in the metastatic setting [13, 45, 46]. This subset of patients represents a possible target for immunotherapy with ICB [47].

\section{Clinical implications}

Defects in specific components of the MMR system can causally induce ET resistance in HR+ breast cancer and thereby, poor patient outcomes [7]. However, MMR deficiency also appears to uncouple hormone signaling and cell cycle regulation at the G1/S cell cycle transition, thereby potentially making these tumors more 


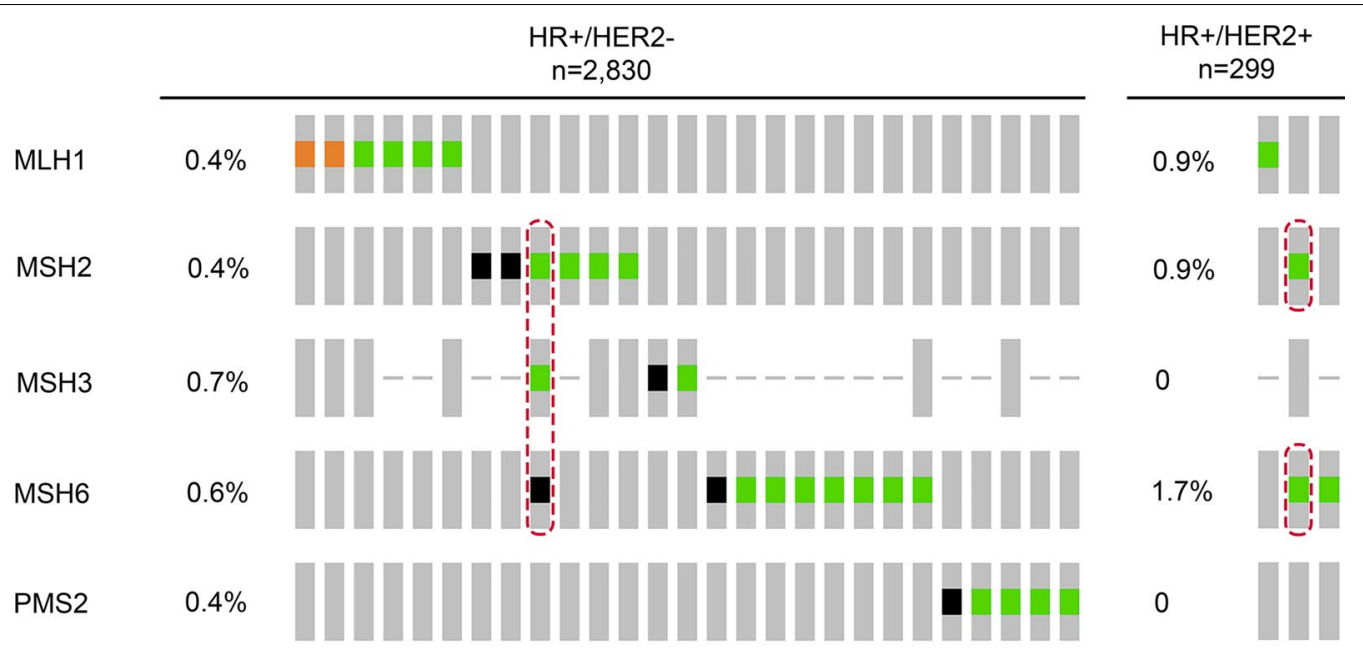

Missense mutation $\quad$ Splice mutation $\quad$ Truncating mutation

Fig. 3 Oncoprint visualization of somatic mutations in the MMR genes acrossHR+/HER2+ and HR+/HER2- breast cancers. Types of alterations are color-coded on thebasis of the legends on the bottom. Each column represents a sample, each rowan MMR gene. Tumors included in this analysis have been retrieved from 14 differentstudies available at cbioportal.org

Table 1 Causes of MMR perturbation at the protein expression level in breast cancer according to the hormone receptor status

\begin{tabular}{lll}
\hline & HR+ (n=69) & HR- (n=12) \\
\hline MutS alone, n (\%) & $17(25)$ & $3(25)$ \\
MSH2 & $4(6)$ & 0 \\
MSH6 & $6(9)$ & 0 \\
Both proteins & & \\
MutL alone, n (\%) & $10(15)$ & $1(8)$ \\
MLH1 & $3(4)$ & 0 \\
PMS2 & $2(3)$ & $1(8)$ \\
Both proteins & & \\
MutS + MutL, n (\%) & $4(6)$ & 0 \\
MutS + MLH1 & $2(3)$ & $1(8)$ \\
MutL+ MSH2 & $1(2)$ & 0 \\
MutL + MSH6 & $8(12)$ & $4(3)$ \\
MSH2 + MLH1 & $1(2)$ & $1(8)$ \\
MSH2 + PMS2 & $2(3)$ & 0 \\
MSH6 + MLH1 & $1(2)$ & 0 \\
MSH6 + PMS2 & $8(12)$ & $1(8)$ \\
All proteins &
\end{tabular}

susceptible to cyclin-dependent kinase (CDK) $4 / 6$ inhibition, even as a front-line therapy [7, 48]. Interestingly, it has been previously reported that a possible mechanism of chemoresistance in HR+/HER2-transformed breast cancer is related to MSH2 downregulation by TGF $\beta$-induced miR-21 [49]. In addition, preclinical data suggest that the DNA homologous recombination gene BRCA1 may be a positive regulator of $\mathrm{MSH} 2$ in $\mathrm{HR}+/$ HER2+breast cancer, suggesting that its tumor-suppressor function can be mediated by the MMR system [50]. In tamoxifen-treated $\mathrm{HR}+$ breast cancer patients, MMR deficiency is related to worse overall and diseasespecific survival (HR 2.29, 95\% CI 1.02-5.17, $p=0.040$ and HR 2.71, 95\% CI 1.00-7.35, $p=0.042$, respectively). This finding suggests a potential role of MMR status to detect $\mathrm{HR}+$ patients who may benefit from treatments other than ET [29]. In another study, the overall survival (OS) rate of dMMR and non-dMMR tumors were profoundly different in both Luminal B and HR- breast cancers. Accordingly, patients with HR+ dMMR carcinomas had worse OS (median $=77$ months, range $=0-115$ months) than those with MMR-proficient (pMMR) or MMR-heterogeneous (hMMR) tumors (median $=84$ months, range $=0-127$ months $)(p=0.008)$. In contrast, HR - dMMR patients treated with chemotherapy showed a better OS compared to the pMMR or hMMR group (median $=87$ and 79 months, range $=73-123$ and 8-113 months, respectively; $p<0.001$ ) [21]. Likewise, the potential effect of each MMR protein has also been studied in different subtypes of breast cancer. In this regard, the most recurrent type of MMR deficiency both in HR+ and HR- breast cancers is related to the loss of expression of $\mathrm{MSH} 2$ alone, with a significant correlation with shorter survival times $(p=0.04)$ [30]. As discussed above, although dMMR colorectal and endometrial 
tumors demonstrate a response to ICB, whether $\mathrm{dMMR}$ breast tumors would do so as well remains untested. This is an interesting question for further study because of the apparent innate differences in the impact of MMR deficiency on TMB in $\mathrm{HR}+$ breast cancers relative to that observed in colorectal or endometrial cancers [37, 51]. A final point of interest to improve actionability against this driver of poor outcomes in $\mathrm{HR}+$ breast cancer is to better understand the biological and functional impact of mutations in MMR genes that are observable in the neoplastic cells. The vast majority of these are individual missense mutations that, at this time, remain variants of unknown significance.

\section{Genetic risk}

The association of germline variants in MLH1, PMS2, MSH2, and MSH6, also referred to as Lynch syndrome genes, with breast cancer risk remains controversial. Unlike in the canonical Lynch-syndrome tumors, MMR deficiency is sporadic in the vast majority of $\mathrm{HR}+$ breast cancer, with only $0.2-0.5 \%$ of cases being classified as syndromic [52]. Consensus in the literature is that there is no statistically significant association between germline mutations in these MMR genes and breast cancer risk [53]. However, some studies do identify an increased risk of breast cancer incidence, and younger age at diagnosis in women with germline variants in PMS2 and MSH6, but no association with germline mutations in MLH1 or MSH2 $[54,55]$. A recent study conducted in a cohort of 711 patients with hereditary breast cancer, reported that $69(9.7 \%)$ patients had at least one germline mutation in the MMR genes. In 32 (4.5\%) of them, these mutations were defined as pathogenic or likely pathogenic [56]. Furthermore, recurrent germline mutations of MLH1 V384D, in the absence of high-TMB, were found in $\sim 14 \%$ of $\mathrm{HR}+/ \mathrm{HER} 2+$ breast cancers in East Asian patients, suggesting that in this subset of neoplasms MLH1 haploinsufficiency is more likely to contribute to tumor predisposition factor rather than to constitute a direct oncogenic driver [57].

\section{Interplay between MMR and the anti-tumor immune response}

There are accumulating data on the interaction between MMR and other immune-related biomarkers that can be employed in the next future to improve the tailored clinical management of HR+ breast cancer. Lately, it has been shown that dMMR breast cancers are related to high tumor-infiltrating lymphocytes (TILs) counts (median of 5, interquartile range 1-10) compared to pMMR tumors (median of $1, p=0.009$ by Mann-Whitney test) [29]. It has been also indicated that dMMR breast cancers show significantly higher expression of programmed death-ligand 1 (PD-L1) and CD8 (69 and 62\%, respectively, $\mathrm{n}=13$ ) than those with intact MMR expression (35 and 29\%, respectively, $n=285$ ) [41]. Another group performed an IHC analysis for CD3, CD4, and CD8 expression on both $\mathrm{HR}+$ and $\mathrm{HR}-$ breast cancers [58]. Interestingly, the Authors observed T-cell predominance together with high TILs in $50 \%(\mathrm{n}=2 / 4) \mathrm{dMMR}$ tumors (range 4-175, per 10 high-power fields). Large multicentric clinical and translational studies specifically designed to include $\mathrm{HR}+$ breast cancers are warranted not only for revealing novel biomarkers but also for better understanding the relationship between MMR and the other traditional biomarkers.

\section{Mismatch repair testing: focus on $\mathrm{HR}+$ breast cancer \\ Rationale}

The pathological identification of dMMR breast cancers has proven to be extremely challenging due to the constraints of the existing methods, and the absence of CDx tests and/or tumor-specific guidelines [12]. So far, the variety of currently available locally developed laboratory tests have been shaped on those approved for colorectal and endometrial carcinomas [59, 60]. For a long time, there has been a nihilistic view of the actual clinical utility of MMR screening for HR+ breast cancer, probably because of the relatively low frequency of the dMMR phenotype in these patients. Lately, the histology-agnostic approval of ICB immunotherapy with pembrolizumab -an anti-programmed cell death-1 (PD-1) drug- for unresectable or metastatic dMMR or MSI-H solid tumors, increased the importance of assessing MMR status in breast cancer [61]. Yet, the likely-predictive and prognostic value of MMR fortifies its role as a promising biomarker to improve clinical decision-making for breast cancer patients [21, 29]. Reference methods for MMR profiling depend on IHC for the four main MMR proteins with or without sequencing assays directed towards selected microsatellite markers (e.g. Bethesda panel and MSI Analysis System) [25, 62]. Despite their reliability, these diagnostic strategies have several limitations, including the relatively low sensitivity in breast cancer due to their heterogeneous protein expression [63-65]. To overcome these issues, new molecular-based methods such as novel real-time PCR (RT-PCR) panels and droplet digital PCR (ddPCR)-based assays, as well as NGS panels have recently emerged (Fig. 4) $[19,66]$.

\section{Immunohistochemistry}

Mutations in MLH1, MSH2, MSH6, and PMS2 may result in proteolysis of the MMR heterodimers and subsequent nuclear loss of protein immunoexpression [67]. Due to its reliability, cost-effectiveness, and the large availability, 


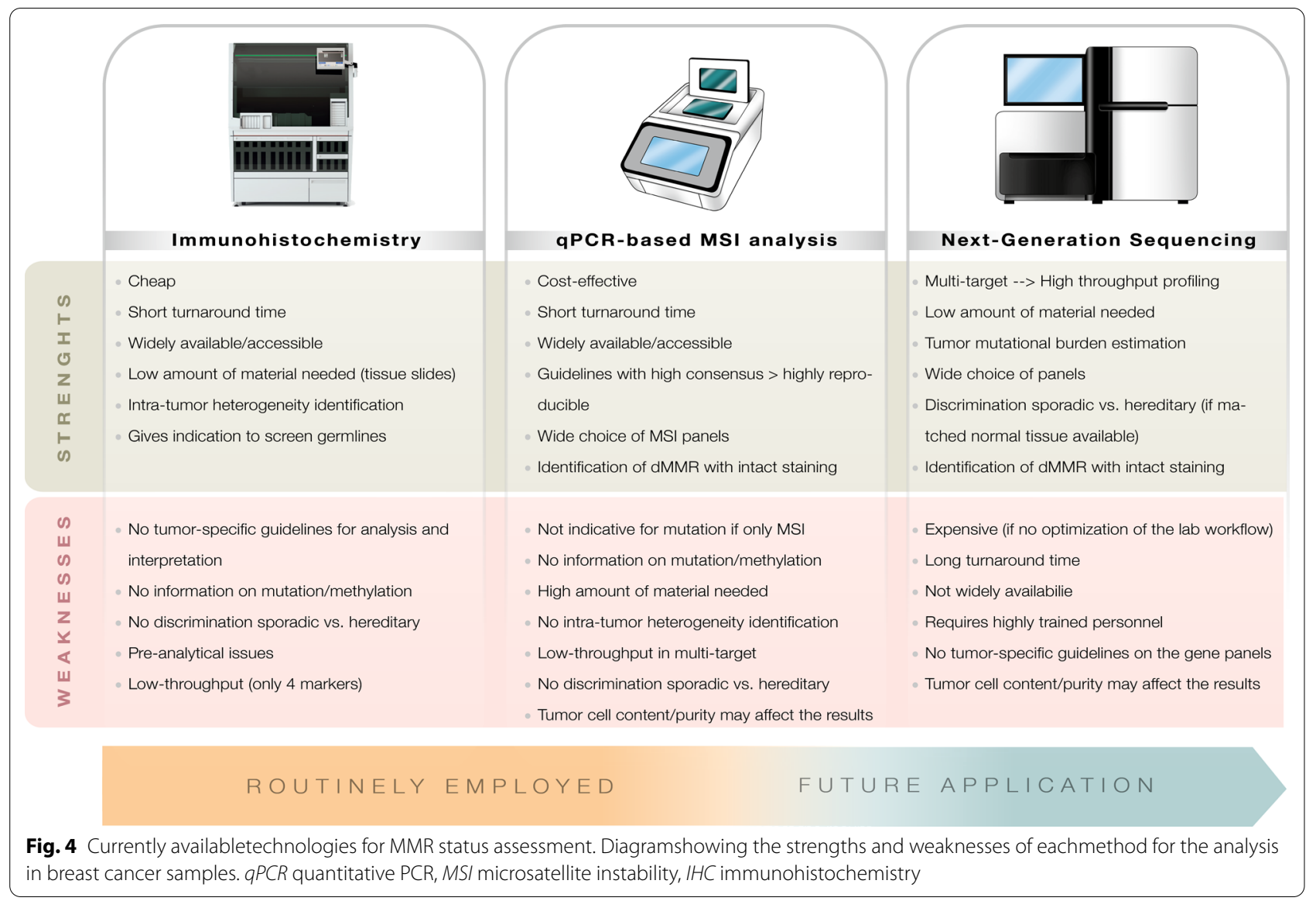

IHC is usually considered a pillar as a first-line MMR testing method [10]. Hence, pre-diluted monoclonal antibodies against MLH1, MSH2, MSH6, and PMS2 are commonly accessible in the vast majority of pathology laboratories [24]. Given the intra-tumor heterogeneity of MMR protein expression in $\mathrm{HR}+$ breast cancer, the reliability and reproducibility of complementary and/or surrogate biomarkers have been investigated by several research groups. Recently, the assessment of phosphatase and tensin homolog (PTEN) expression, a key tumor suppressor gene mainly involved in DNA repair, apoptosis, and cell survival, was suggested as a complementary biomarker to pre-screen MMR status in breast cancer $[30,68]$. According to this workflow, the positive predictive value of PTEN retained status for pMMR ranged from $94.6 \%$ in HR+tumors to $100 \%$ in HER2-enriched and HR - breast cancers. Moreover, a significant association of the MMR status with PTEN IHC was seen in the HR+ cluster $(p<0.0001$, Fisher's exact test) suggesting that alterations in PTEN expression are significantly related to MMR deficiency [69]. However, it has been recently demonstrated that, in breast cancer, MMR protein loss is more frequently detected compared to MSI, suggesting a lack of interchangeability of these two tests [21]. Importantly, not all MMR proteins evenly influence either mutational load or MSI when deficient [30, 70]. The potential impact of technical artifacts and/or intratumor heterogeneity phenomena on MMR status assessment still needs to be fully elucidated [71]. Albeit MMR IHC in breast cancer is being suggested as a valid clinical test by different studies, several issues including the use of specific antibody clones, CDx, and/or interpretation guidelines have still to be addressed. Other major drawbacks of this technique are related to the lack of specific recommendations on cold ischemia time, fixation protocols, primary antibody clones, concentrations, and staining platform as well as interpretation guidelines [72].

\section{qPCR-based MSI analysis}

MSI analysis has been originally performed by RT-PCR for five microsatellite markers, consisting of three dinucleotides (i.e. D2S123, D5S346, and D17S250) and two mononucleotides (i.e. BAT-25 and BAT-26) repeats [7375]. This panel, however, as recommended by the revised Bethesda Guidelines, has proven sufficiently high sensitivity and specificity only in the detection of syndromic 
tumors [76, 77]. Novel PCR panels (e.g. MSI Analysis System, Promega ${ }^{\circledR}$ ) targeting mononucleotide repeats have been proposed as reliable alternative options to the Bethesda one [78]. Lately, new high-performance assays have been proposed as viable and complementary options to IHC and standard RT-PCR panels, including PlentiPlex $^{\mathrm{TM}}$ MSI (Pentabase), OncoMate ${ }^{\mathrm{TM}}$ (Promega), Idylla ${ }^{\mathrm{TM}}$ MSI Test (Biocartis), TrueMark (Thermofisher), and BioRad ddPCR [79]. However, no data are currently available for their reliability in breast cancer. The major limitation of RT-PCR assays is that insufficient tumor content may not allow the detection of MSI and/or alterations in the sequence target. Furthermore, in tumors with low MSI/ dMMR frequency, such as breast cancer, little data are available and the exploitation of IHC and MSI RT-PCR protocols is highly questioned [12]. Of note, epigenetic silencing of MLH1 by promoter hypermethylation is a crucial event that may lead to MMR deficiency. Methylation-specific PCR for the $5^{\prime}$ CpG promoter of MLH1 is primarily carried out for the assessment of this condition [80].

\section{Next-generation sequencing}

Recently, NGS has appeared as an ultra-sensitive method to characterize MSI and MMR status accurately and simultaneously [81]. Thereby, NGS-based methods demonstrated higher performances when compared to previous technologies and are potentially useful to expand MSI testing, particularly in those cancers characterized by lower MSI-H/dMMR frequencies [82]. Indeed, NGS panels can screen a larger number of microsatellite loci compared to RT-PCR [83]. This allows parallel high-throughput analysis of both microsatellites and genes leading to the simultaneous identification of other actionable alterations. Interestingly, MSI testing performed using NGS can be easily integrated with other relevant biomarkers such as TMB, using targeted-specific panels and avoiding the costs of whole-exome or whole-genome sequencing $[84,85]$. To date, NGS is rarely performed in breast cancer due to its higher cost compared to lower throughput methods, and the lack of tumor-specific panels of genes which could reveal a potential association of MMR deficiency with other clinically actionable genes [66]. It is important to keep in mind that current NGS genomic diagnoses of MMR deficiency through TMB, genomic scars, or MSI were developed based on canonical Lynch syndrome cancers (colorectal predominantly) [86]. To ensure high specificity and sensitivity, these diagnostic strategies might need to be re-developed in the context of breast cancer. Considering all the pros and cons, although the aforementioned methods represent candidate tools for the establishment of promising MMR testing strategies, they require profound experimentation before being implemented in clinical setting.

\section{Concluding remarks}

Alterations of the MMR system are relatively rare events in $\mathrm{HR}+$ breast cancer. However, due to the extremely high frequency of this neoplastic condition in the female population worldwide, MMR deficiency is of great clinical interest. The prognostic value of MMR deficiency coupled with its predictive role for ICB and ET resistance further increases the significance of performing this analysis in HR+ breast cancers. From the diagnostic perspective, it is crucial to adopt a tailored methodology to cover all of the intrinsic characteristics of breast cancers that are usually not shared by other types of tumors where MMR deficiency is more common. The versatility and efficiency of RT-PCR, combined with its cost-effectiveness, sensitivity, and specificity, facilitate the adoption of this technology in virtually all molecular pathology laboratories. On the other hand, NGS panels allow covering several different alterations simultaneously, even starting from low input DNA. Although the rough costs of this technology, which requires specialized centers with highly trained personnel, are relatively high, the optimization of the laboratory workflow allows for a favorable cost-benefit ratio. On the other hand, pathologists, molecular biologists, and clinicians should be fully aware of the lack of interchangeability between MMR protein expression profiling and gene sequencing in HR+ breast cancers. It is important to remark that in the case of negative results at DNA analysis, a proteomic technique should be performed to avoid false-negative results. Further translational research and clinical studies coupled with tumor-specific guidelines for analytical and preanalytical phases are warranted to precisely characterize MMR status in $\mathrm{HR}+$ breast cancer.

\section{Abbreviations \\ HR: Hormone receptor; MMR: Mismatch repair; ICB: Immune checkpoint block- ers; CDx: Companion diagnostic tests; dMMR: MMR-deficient; MLH1: MutL homolog 1; MSH2: MutS homolog 2; MSH6: MutS homolog 6; MSH3: MutS homolog 3; PMS2: Post-meiotic segregation increased 2; PCNA: Proliferating cellular nuclear antigen; MSI: Microsatellite instability; MSI-H: MSI-high; TMB: Tumor mutation burden; NGS: Next-generation sequencing; PI3K: Phospho- inositide 3 kinase; mTOR: Mammalian target of rapamycin; HLA: Human leukocyte antigen; CDK: Cyclin-dependent kinase; OS: Overall survival; pMMR: MMR-proficient; hMMR: MMR-heterogeneous; TILs: Tumor-infiltrating lym- phocytes; PD-1: Programmed cell death-1; PD-L1: Programmed death-ligand 1; IHC: Immunohistochemistry; RT-PCR: Real-time PCR; ddPCR: Droplet digital PCR; PTEN: Phosphatase and tensin homolog.}

\section{Acknowledgements}

The authors would like to thank Mrs. Erika Pizzo for the graphic development of the figures. 


\section{Authors' contributions}

ES and KV contributed to the literature search, conception and design of the article and drafted the first version of the manuscript. RP, MI, and EGR. contributed to literature search and provided critical revisions of the manuscript. $\mathrm{SH}$ contributed to literature search, design of the article and provided critical revisions of the manuscript. NF contributed to conception and design of the article and provided critical revisions of the manuscript. All the authors provided final approval to the submitted work. All authors read and approved the final manuscript.

\section{Funding}

$\mathrm{SH}$ is supported by the National Cancer Institute of the National Institutes of Health under Award Number CA229613. The content is solely the responsibility of the authors and does not necessarily represent the official views of the National Institutes of Health.

\section{Availability of data and materials}

Not applicable.

\section{Declarations}

\section{Ethics approval and consent to participate} Not applicable.

\section{Consent for publication}

Not applicable.

\section{Competing interests}

M.I. received honoraria for consulting/advisory role/speaker bureau from Errekappa Euroterapici S.p.a. E.G.R. has received advisory fees from Novartis, Roche, and MSD Italia; honoraria from Thermo Fisher Scientific, AstraZeneca, Roche. S.H. has two provisional patent licenses outlining a role for DNA repair defects in predicting response to therapies in breast cancer patients (Serial Nos: 62/670,368, 63/106777). N.F. has received honoraria for consulting, advisory role, and/or speaker bureau from Merck Sharp \& Dohme (MSD), Boehringer Ingelheim, and Novartis. These companies had no role in the design of the study; in the collection, analyses, or interpretation of data; in the writing of the manuscript, and/or in the decision to publish the results. All other authors declare that they have no competing interests.

\section{Author details}

${ }^{1}$ Division of Pathology, IEO, European Institute of Oncology IRCCS, University of Milan, Via Giuseppe Ripamonti 435, 20141 Milan, Italy. ${ }^{2}$ Department of Oncology and Hemato-Oncology, University of Milan, Via Festa del Perdono 7, 20122 Milan, Italy. ${ }^{3}$ Physical and Rehabilitative Medicine, Department of Health Sciences, University of Eastern Piedmont, Viale Piazza D'Armi, 1, 28100 Novara, Italy. ${ }^{4}$ Department of Tumor Microenvironment and Cancer Immunology, Sanford Burnham Prebys Medical Discovery Institute, $10901 \mathrm{~N}$ Torrey Pines Rd, 92037 La Jolla, CA, USA.

Received: 5 March 2021 Accepted: 7 May 2021

Published online: 17 May 2021

\section{References}

1. Heer E, Harper A, Escandor N, Sung H, McCormack V, Fidler-Benaoudia MM. Global burden and trends in premenopausal and postmenopausal breast cancer: a population-based study. Lancet Glob Health. 2020;8(8):e1027-37.

2. Lopez G, Costanza J, Colleoni M, Fontana L, Ferrero S, Miozzo M, Fusco N. Molecular insights into the classification of luminal breast cancers: the genomic heterogeneity of progesterone-negative tumors. Int J Mol Sci. 2019;20(3):510.

3. Lei JT, Anurag M, Haricharan S, Gou X, Ellis MJ. Endocrine therapy resistance: new insights. Breast. 2019;48(1):S26-30.

4. Huang H, Zhou J, Chen H, Li J, Zhang C, Jiang X, Ni C. The immunomodulatory effects of endocrine therapy in breast cancer. J Exp Clin Cancer Res. 2021;40(1):19.
5. Anurag M, Punturi N, Hoog J, Bainbridge MN, Ellis MJ, Haricharan S. Comprehensive profiling of DNA repair defects in breast cancer identifies a novel class of endocrine therapy resistance drivers. Clin Cancer Res. 2018;24(19):4887-99.

6. Grizzi G, Ghidini M, Botticelli A, Tomasello G, Ghidini A, Grossi F, Fusco N, Cabiddu M, Savio T, Petrelli F. Strategies for increasing the effectiveness of aromatase inhibitors in locally advanced breast cancer: an evidencebased review on current options. Cancer Manag Res. 2020;12:675-86.

7. Haricharan S, Punturi N, Singh P, Holloway KR, Anurag M, Schmelz J, Schmidt C, Lei JT, Suman V, Hunt K, et al. Loss of MutL disrupts CHK2dependent cell-cycle control through cdk4/6 to promote intrinsic endocrine therapy resistance in primary breast cancer. Cancer Discov. 2017;7(10):1168-83.

8. Pećina-Šlaus N, Kafka A, Salamon I, Bukovac A. Mismatch repair pathway, genome stability and cancer. Front Mol Biosci. 2020;7:122-122.

9. Liu D, Keijzers G, Rasmussen LJ. DNA mismatch repair and its many roles in eukaryotic cells. Mutat Res. 2017;773:174-87.

10. Corti C, Sajjadi E, Fusco N. Determination of mismatch repair status in human cancer and its clinical significance: does one size fit all? Adv Anat Pathol. 2019;26(4):270-9.

11. Pagni F, Guerini-Rocco E, Schultheis AM, Grazia G, Rijavec E, Ghidini M, Lopez G, Venetis K, Croci GA, Malapelle U, et al. Targeting immune-related biological processes in solid tumors: we do need biomarkers. Int J Mol Sci. 2019;20(21):5452.

12. Venetis K, Sajjadi E, Haricharan S, Fusco N. Mismatch repair testing in breast cancer: the path to tumor-specific immuno-oncology biomarkers. Translational Cancer Research. 2020. https://doi.org/10.21037/ tcr-20-1852.

13. Heeke AL, Xiu J, Elliott A, Korn WM, Lynce F, Pohlmann PR, Isaacs C, Swain SM, Vidal G, Schwartzberg LS, et al. Actionable co-alterations in breast tumors with pathogenic mutations in the homologous recombination DNA damage repair pathway. Breast Cancer Res Treat. 2020;184(2):265-75.

14. Eso Y, Shimizu T, Takeda H, Takai A, Marusawa H. Microsatellite instability and immune checkpoint inhibitors: toward precision medicine against gastrointestinal and hepatobiliary cancers. J Gastroenterol. 2020;55(1):15-26.

15. Zhang $L$, Peng $Y$, Peng G. Mismatch repair-based stratification for immune checkpoint blockade therapy. Am J Cancer Res. 2018;8(10):1977-88.

16. Huang Y, Li GM. DNA mismatch repair preferentially safeguards actively transcribed genes. DNA Repair (Amst). 2018;71:82-6.

17. Tamura K, Kaneda M, Futagawa M, Takeshita M, Kim S, Nakama M, Kawashita N, Tatsumi-Miyajima J. Genetic and genomic basis of the mismatch repair system involved in Lynch syndrome. Int J Clin Oncol. 2019;24(9):999-1011.

18. Watkins JA, Irshad S, Grigoriadis A, Tutt AN. Genomic scars as biomarkers of homologous recombination deficiency and drug response in breast and ovarian cancers. Breast cancer research : BCR. 2014;16(3):211.

19. Piciotti R, Venetis K, Sajjadi E, Fusco N. Mismatch repair status characterization in oncologic pathology: taking stock of the real-world possibilities. Journal of Molecular Pathology. 2021;2(2):93-100.

20. Li K, Luo H, Huang L, Luo H, Zhu X. Microsatellite instability: a review of what the oncologist should know. Cancer Cell Int. 2020;20(1):16.

21. Fusco N, Lopez G, Corti C, Pesenti C, Colapietro P, Ercoli G, Gaudioso G, Faversani A, Gambini D, Michelotti A, et al. Mismatch repair protein loss as a prognostic and predictive biomarker in breast cancers regardless of microsatellite instability. JNCI Cancer Spectr. 2018;2(4):pky056.

22. Bagshaw ATM. Functional mechanisms of microsatellite DNA in eukaryotic genomes. Genome Biol Evol. 2017;9(9):2428-43.

23. Garrido-Ramos MA. Satellite DNA: An evolving topic. Genes. 2017;8(9):230.

24. Shia J. The diversity of tumours with microsatellite instability: molecular mechanisms and impact upon microsatellite instability testing and mismatch repair protein immunohistochemistry. Histopathology. 2020;78(4):485-97.

25. Sajjadi E, Venetis K, Scatena C, Fusco N. Biomarkers for precision immunotherapy in the metastatic setting: hope or reality? Ecancermedicalscience. 2020;14:1150.

26. Mills AM, Dill EA, Moskaluk CA, Dziegielewski J, Bullock TN, Dillon PM. The relationship between mismatch repair deficiency and PD-L1 expression in breast carcinoma. Am J Surg Pathol. 2018;42(2):183-91. 
27. Pereira B, Chin SF, Rueda OM, Vollan HK, Provenzano E, Bardwell HA, Pugh $M$, Jones $L$, Russell R, Sammut SJ, et al. The somatic mutation profiles of 2,433 breast cancers refines their genomic and transcriptomic landscapes. Nat Commun. 2016;7:11479.

28. Anurag M, Ellis MJ, Haricharan S. DNA damage repair defects as a new class of endocrine treatment resistance driver. Oncotarget. 2018;9(91):36252-3.

29. Cheng AS, Leung SCY, Gao D, Burugu S, Anurag M, Ellis MJ, Nielsen TO. Mismatch repair protein loss in breast cancer: clinicopathological associations in a large British Columbia cohort. Breast Cancer Res Treat. 2020;179(1):3-10.

30. Lopez G, Noale M, Corti C, Gaudioso G, Sajjadi E, Venetis K, Gambini D, Runza L, Costanza J, Pesenti C, et al. PTEN expression as a complementary biomarker for mismatch repair testing in breast cancer. Int J Mol Sci. 2020;21(4):1461.

31. Helleday T, Eshtad S, Nik-Zainal S. Mechanisms underlying mutational signatures in human cancers. Nat Rev Genet. 2014;15(9):585-98.

32. Dasgupta H, Islam S, Alam N, Roy A, Roychoudhury S, Panda CK. Hypomethylation of mismatch repair genes $\mathrm{MLH} 1$ and $\mathrm{MSH} 2$ is associated with chemotolerance of breast carcinoma: Clinical significance. J Surg Oncol. 2019;119(1):88-100.

33. Viale G, Trapani D, Curigliano G. Mismatch repair deficiency as a predictive biomarker for immunotherapy efficacy. Biomed Res Int. 2017;2017:4719194

34. Boyiadzis MM, Kirkwood JM, Marshall JL, Pritchard CC, Azad NS, Gulley $\mathrm{J}$. Significance and implications of FDA approval of pembrolizumab for biomarker-defined disease. J Immunother Cancer. 2018;6(1):35.

35. Subbiah $V$, Kurzrock $R$. The marriage between genomics and immunotherapy: mismatch meets its match. Oncologist. 2019;24(1):1-3.

36. Le DT, Durham JN, Smith KN, Wang H, Bartlett BR, Aulakh LK, Lu S, Kemberling $\mathrm{H}$, Wilt C, Luber BS, et al. Mismatch repair deficiency predicts response of solid tumors to PD-1 blockade. Science. 2017;357(6349):409-13.

37. Haricharan S, Bainbridge MN, Scheet P, Brown PH. Somatic mutation load of estrogen receptor-positive breast tumors predicts overall survival: an analysis of genome sequence data. Breast Cancer Res Treat. 2014;146(1):211-20.

38. Barroso-Sousa R, Jain E, Cohen O, Kim D, Buendia-Buendia J, Winer E, Lin $\mathrm{N}$, Tolaney SM, Wagle N. Prevalence and mutational determinants of high tumor mutation burden in breast cancer. Ann Oncol. 2020;31 (3):387-94.

39. Lopez G, Fusco N. Mismatch repair protein loss in breast cancer: clinicopathological associations in a large British Columbia cohort. Breast Cancer Res Treat. 2020. https://doi.org/10.1007/s10549-10020-05530-10548.

40. Lopez G, Venetis K, Sajjadi E, Fusco N. Mismatch repair system genomic scars in gastroesophageal cancers: biology and clinical testing. Gastrointestinal Disorders. 2020;2(4):341-52.

41. Hou Y, Nitta H, Parwani AV, Li Z. PD-L1 and CD8 are associated with deficient mismatch repair status in triple-negative and HER2-positive breast cancers. Hum Pathol. 2019:86:108-14.

42. Smith J, Tho LM, Xu N, Gillespie DA. The ATM-Chk2 and ATR-Chk1 pathways in DNA damage signaling and cancer. Adv Cancer Res. 2010;108:73-112.

43. Vasan N, Toska E, Scaltriti M. Overview of the relevance of PI3K pathway in HR-positive breast cancer. Ann Oncol. 2019;30(Suppl 10):x3-11.

44. Fusco N, Malapelle U, Fassan M, Marchiò C, Buglioni S, Zupo S, Criscitiello C, Vigneri P, Dei Tos AP, Maiorano E. PIK3CA mutations as a molecular target for hormone receptor-positive, HER2-negative metastatic breast cancer. Front Oncol. 2021;11:562.

45. Kawaji H, Kubo M, Yamashita N, Yamamoto H, Kai M, Kajihara A, Yamada M, Kurata K, Kaneshiro K, Harada Y, et al. Comprehensive molecular profiling broadens treatment options for breast cancer patients. Cancer Med. 2020;10(2):529-39.

46. Garrido-Castro AC, Spurr LF, Hughes ME, Li YY, Cherniack AD, Kumari P, Lloyd MR, Bychkovsky B, Barroso-Sousa R, Di Lascio S, et al. Genomic characterization of de novo metastatic breast cancer. Clin Cancer Res. 2020;27(4):1105-18.

47. Benvenuto M, Focaccetti C, Izzi V, Masuelli L, Modesti A, Bei R. Tumor antigens heterogeneity and immune response-targeting neoantigens in breast cancer. Semin Cancer Biol. 2019. https://doi.org/10.1016/j.semca ncer.2019.10.023.
48. Anurag M, Haricharan S, Ellis MJ. CDK4/6 Inhibitor biomarker research: are we barking up the wrong tree? Clin Cancer Res. 2020;26(1):3-5.

49. Yu Y, Wang Y, Ren X, Tsuyada A, Li A, Liu LJ, Wang SE. Context-dependent bidirectional regulation of the MutS homolog 2 by transforming growth factor $\beta$ contributes to chemoresistance in breast cancer cells. Mol Cancer Res. 2010;8(12):1633-42.

50. Atalay A, CrookT, Ozturk M, Yulug IG. Identification of genes induced by BRCA1 in breast cancer cells. Biochem Biophys Res Commun. 2002;299(5):839-46.

51. Krug K, Jaehnig EJ, Satpathy S, Blumenberg L, Karpova A, Anurag M, Miles G, Mertins P, Geffen Y, Tang LC, et al. Proteogenomic landscape of breast cancer tumorigenesis and targeted therapy. Cell. 2020;183(5):1436-1456. e1431.

52. de la Chapelle A. The incidence of Lynch syndrome. Fam Cancer. 2005;4(3):233-7.

53. Ten Broeke SW, van der Klift HM, Tops CMJ, Aretz S, Bernstein I, Buchanan DD, de la Chapelle A, Capella G, Clendenning M, Engel C, et al. Cancer risks for PMS2-associated lynch syndrome. J Clin Oncol. 2018;36(29):2961-8.

54. Roberts ME, Jackson SA, Susswein LR, Zeinomar N, Ma X, Marshall ML, Stettner AR, Milewski B, Xu Z, Solomon BD, et al. MSH6 and PMS2 germline pathogenic variants implicated in Lynch syndrome are associated with breast cancer. Genetics Med. 2018;20(10):1167-74.

55. Sheehan M, Heald B, Yanda C, Kelly ED, Grobmyer S, Eng C, Kalady M, Pederson $\mathrm{H}$. Investigating the link between lynch syndrome and breast cancer. Eur J Breast Health. 2020;16(2):106-9.

56. Nikitin AG, Chudakova DA, Enikeev RF, Sakaeva D, Druzhkov M, Shigapova LH, Brovkina Ol, Shagimardanova El, Gusev OA, Gordiev MG. Lynch syndrome germline mutations in breast cancer: next generation sequencing case-control study of 1,263 participants. Front Oncol. 2020;10:666.

57. Lee SE, Lee HS, Kim KY, Park JH, Roh H, Park HY, Kim WS. High prevalence of the MLH1 V384D germline mutation in patients with HER2-positive luminal B breast cancer. Sci Rep. 2019;9(1):10966.

58. Wen YH, Brogi E, Zeng Z, Akram M, Catalano J, Paty PB, Norton L, Shia J. DNA mismatch repair deficiency in breast carcinoma: a pilot study of triple-negative and non-triple-negative tumors. Am J Surg Pathol. 2012;36(11):1700-8.

59. Loughrey MB, McGrath J, Coleman HG, Bankhead P, Maxwell P, McGready C, Bingham V, Humphries MP, Craig SG, McQuaid S, et al. Identifying mismatch repair-deficient colon cancer: near-perfect concordance between immunohistochemistry and microsatellite instability testing in a large, population-based series. Histopathology. 2020;78(3):401-13.

60. Stelloo E, Jansen AML, Osse EM, Nout RA, Creutzberg CL, Ruano D, Church DN, Morreau H, Smit V, van Wezel T, et al. Practical guidance for mismatch repair-deficiency testing in endometrial cancer. Ann Oncol. 2017:28(1):96-102.

61. Lemery S, Keegan P, Pazdur R. First FDA approval agnostic of cancer site - when a biomarker defines the indication. N Engl J Med. 2017;377(15):1409-12.

62. Sahin IH, Akce M, Alese O, Shaib W, Lesinski GB, El-Rayes B, Wu C. Immune checkpoint inhibitors for the treatment of MSI-H/MMR-D colorectal cancer and a perspective on resistance mechanisms. Br J Cancer. 2019;121(10):809-18

63. LuY, Soong TD, Elemento O. A novel approach for characterizing microsatellite instability in cancer cells. PLOS ONE. 2013;8(5):e63056.

64. Carethers JM. Microsatellite instability pathway and EMAST in colorectal cancer. Current Colorectal Cancer Rep. 2017;13(1):73-80.

65. Poulogiannis G, Frayling IM, Arends MJ. DNA mismatch repair deficiency in sporadic colorectal cancer and Lynch syndrome. Histopathology. 2010;56(2):167-79.

66. Luchini C, Bibeau F, Ligtenberg MJL, Singh N, Nottegar A, Bosse T, Miller R, Riaz N, Douillard JY, Andre F, et al. ESMO recommendations on microsatellite instability testing for immunotherapy in cancer, and its relationship with PD-1/PD-L1 expression and tumour mutational burden: a systematic review-based approach. Ann Oncol. 2019;30(8):1232-43.

67. Kosinski J, Hinrichsen I, Bujnicki JM, Friedhoff P, Plotz G. Identification of Lynch syndrome mutations in the MLH1-PMS2 interface that disturb dimerization and mismatch repair. Hum Mutat. 2010;31 (8):975-82.

68. Alowiri NH, Hanafy SM, Haleem RA, Abdellatif A. PIK3CA and PTEN genes expressions in breast cancer. Asian Pac J Cancer Prev. 2019;20(9):2841-6. 
69. Fusco N, Sajjadi E, Venetis K, Gaudioso G, Lopez G, Corti C, Rocco EG, Criscitiello C, Malapelle U, Invernizzi M. PTEN alterations and their role in cancer management: are we making headway on precision medicine? Genes (Basel). 2020;11(7):719.

70. McCarthy AJ, Capo-Chichi JM, Spence T, Grenier S, Stockley T, Kamel-Reid S, Serra S, Sabatini P, Chetty R. Heterogenous loss of mismatch repair (MMR) protein expression: a challenge for immunohistochemical interpretation and microsatellite instability (MSI) evaluation. J Pathol Clin Res. 2019:5(2):115-29.

71. Horimoto Y, Thinzar Hlaing M, Saeki H, Kitano S, Nakai K, Sasaki R, KurisakiArakawa A, Arakawa A, Otsuji N, Matsuoka S, et al. Microsatellite instability and mismatch repair protein expressions in lymphocyte-predominant breast cancer. Cancer Sci. 2020;111(7):2647-54.

72. Bussolati G, Annaratone L, Maletta F. The pre-analytical phase in surgical pathology. Recent Results Cancer Res. 2015;199:1-13.

73. Umar A, Boland CR, Terdiman JP, Syngal S, de la Chapelle A, Rüschoff J, Fishel R, Lindor NM, Burgart LJ, Hamelin R, et al. Revised Bethesda Guidelines for hereditary nonpolyposis colorectal cancer (Lynch syndrome) and microsatellite instability. J Natl Cancer Inst. 2004;96(4):261-8.

74. Movahedi-Lankara S, Gilks B, Soslow RA, Christopher NO, Oliva E: Protocol for the examination of specimens from patients with carcinoma of the endometrium. In:: College of American Pathologists; October 2013.

75. Rodriguez-Bigas MA, Boland CR, Hamilton SR, Henson DE, Jass JR, Khan PM, Lynch H, Perucho M, Smyrk T, Sobin L, et al. A National Cancer Institute Workshop on Hereditary Nonpolyposis Colorectal Cancer Syndrome: meeting highlights and Bethesda guidelines. J Natl Cancer Inst. 1997;89(23):1758-62.

76. Gilson P, Levy J, Rouyer M, Demange J, Husson M, Bonnet C, Salleron J, Leroux A, Merlin J-L, Harlé A. Evaluation of 3 molecular-based assays for microsatellite instability detection in formalin-fixed tissues of patients with endometrial and colorectal cancers. Sci Rep. 2020;10(1):16386.

77. Boland CR, Thibodeau SN, Hamilton SR, Sidransky D, Eshleman JR, Burt RW, Meltzer SJ, Rodriguez-Bigas MA, Fodde R, Ranzani GN, et al. A National Cancer Institute Workshop on Microsatellite Instability for cancer detection and familial predisposition: development of international criteria for the determination of microsatellite instability in colorectal cancer. Cancer Res. 1998;58(22):5248-57.
78. Zeinalian M, Hashemzadeh-Chaleshtori M, Salehi R, Emami MH. Clinical aspects of microsatellite instability testing in colorectal cancer. Adv Biomed Res. 2018;7:28-28.

79. De Craene B, Van de Velde J, Rondelez E, Vandenbroeck L, Peeters K, Vanhoey T, Elsen N, Vandercruyssen G, Melchior L, Willemoe GL, et al. Detection of microsatellite instability (MSI) in colorectal cancer samples with a novel set of highly sensitive markers by means of the Idylla MSI Test prototype. J Clin Oncol . 2018;36(15):15639.

80. Li K, Luo H, Huang L, Zhu X. Microsatellite instability: a review of what the oncologist should know. Cancer Cell Int. 2020;20:16.

81. Salipante SJ, Scroggins SM, Hampel HL, Turner EH, Pritchard CC. Microsatellite instability detection by next generation sequencing. Clin Chem. 2014;60(9):1192-9.

82. Middha S, Zhang L, Nafa K, Jayakumaran G, Wong D, Kim HR, Sadowska J, Berger MF, Delair DF, Shia J et al: Reliable pan-cancer microsatellite instability assessment by using targeted next-generation sequencing data. JCO Precis Oncol 2017, 2017.

83. Bonneville R, Krook MA, Chen H-Z, Smith A, Samorodnitsky E, Wing MR, Reeser JW, Roychowdhury S. Detection of microsatellite instability biomarkers via next-generation sequencing. Methods Mol Bio (Clifton, $\mathrm{NJ}$ ). 2020;2055:119-32.

84. Albayrak A, Garrido-Castro AC, Giannakis M, Umeton R, Manam MD, Stover EH, Porter RL, Johnson BE, Liaw K-L, Amonkar M, et al. Clinical pan-cancer assessment of mismatch repair deficiency using tumoronly Targeted Next-Generation Sequencing. JCO Precision Oncol. 2020;4:1084-97.

85. Angerilli V, Galuppini F, Pagni F, Fusco N, Malapelle U, Fassan M. The role of the pathologist in the next-generation era of tumor molecular characterization. Diagnostics. 2021;11(2):339.

86. Kucab JE, Zou X, Morganella S, Joel M, Nanda AS, Nagy E, Gomez C, Degasperi A, Harris R, Jackson SP, et al. A compendium of mutational signatures of environmental agents. Cell. 2019;177(4):821-836.e816.

\section{Publisher's Note}

Springer Nature remains neutral with regard to jurisdictional claims in published maps and institutional affiliations.
Ready to submit your research? Choose BMC and benefit from:

- fast, convenient online submission

- thorough peer review by experienced researchers in your field

- rapid publication on acceptance

- support for research data, including large and complex data types

- gold Open Access which fosters wider collaboration and increased citations

- maximum visibility for your research: over 100M website views per year

At BMC, research is always in progress.

Learn more biomedcentral.com/submissions 